\title{
EXPLORING THE EVOLUTIONARY HISTORY OF A GROUP USING MULTIPLE MORPHOSPACES OF VARYING COMPLEXITY AND PHILOSOPHY
}

CHAPMAN*, Ralph E., Applied Morphometrics Laboratory, ADP NHB MRC 136, National Museum of Natural History, Smithsonian Institution, Washington, DC 20560, U.S.A.; RASSKIN-GUTMAN, Diego, Unidad de Paleontologia, Universidad Autonoma de Madrid, Spain; WEISHAMPEL, David B., Department of Cell Biology and Anatomy, The Johns Hopkins University School of Medicine, Baltimore, MD 21205, U.S.A.

Morphospace models have been of interest to paleobiologists for more than 30 years and have seen varying numbers of applications to different taxonomic groups with varying levels of success. As noted by George McGhee, two types of morphospaces have been described in the literature; theoretical and empirical. Theoretical morphospaces are constructed on the basis of a priori assumptions about theoretical morphology - the morphospace exists without the organisms being studied, which are then fit into it. Classic "Raupian" morphospace studies on shell coiling parameters are examples. Empirical morphospaces, on the other hand, are developed in the opposite direction. Large numbers of organisms are analyzed morphometrically and the morphospace developed using these data, typically through the application of ordination methods. Many recent papers on trilobites, echinoderms and molluscs have used this latter approach. Not surprisingly, theoretical morphospaces typically work from a less complex description of morphology than many empirical studies can, although the interpretation of the biological meaning of very complex, empirical studies can be difficult. There are ways to use both approaches to maximize the information derived from morphospace studies.

We will demonstrate that, for the study of a single group of organisms, there is a great utility in developing morphospace models that conform to both the theoretical and empirical models. Further, we suggest the development of a series of progressively more complex morphospaces, allowing the results from the simpler morphospaces to help develop the more and more complex ones. In this way, the complex results can be much more easily interpreted in the light of the patterns seen from the less complex studies and in ways more relevant to the biology of the organisms being studied. For examples we will develop morphospace models for limb proportions in tetrapods and cephalon shapes in trilobites.

Finally, we will discuss the utility of certain morphometric methods, such as Fourier analysis, for developing morphospaces. Contrary to some reports, such methods can be used to develop both theoretical and empirical morphospaces. Further, these methods also can be used to develop morphospaces of varying complexity as well. 